\title{
Automated Geochemical and Mineralogical Synchrotron Characterization of Bulk Geological Materials: an Innovation for the Minerals Industry.
}

\author{
Neil R. Banerjee $^{1 *}$, Lisa L. Van Loon ${ }^{1,2}$, Ramjay J. Botor ${ }^{1}$, and Trevor J. Flynn ${ }^{1}$ \\ 1. Department of Earth Sciences, Western University, London, Canada. \\ 2. LISA CAN Analytical Solutions Inc., Saskatoon, Canada. \\ * Corresponding author, neil.banerjee@uwo.ca
}

Introduction: In the minerals industry, a cornerstone of the Canadian economy, innovation is the key to success. Synchrotron X-ray diffraction is now a routine analysis in the pharmaceutical industry and has pushed the development of automated sample handling to remotely collect high quality data with rapid throughput. The application of this rapid, high quality, high resolution, and high throughput analysis represents a paradigm shift for the minerals industry. The high flux available from a synchrotron source provides a faster, higher quality analysis for hundreds and even thousands of samples - something that is not possible with laboratory instruments.

X-ray diffraction is a time-proven tool to decipher the mineralogical make up of geological materials, mineral processing residues, and mine wastes. X-ray fluorescence is used to determine the chemical composition of complex materials, with sensitivity in the parts-per-million. By combining synchrotron radiation powder X-ray diffraction (SR-XRD) and X-ray fluorescence (SR-XRF), a richer, more complete characterization of these complex materials is developed. The addition of X-ray Absorption Near Edge Structure (XANES) spectroscopy allows for further understanding by targeting the chemical speciation and oxidation states of elements of interest like gold and arsenic. The creation of large data sets will allow the application of advanced analytical computing techniques that are becoming commonplace in a wide variety of industry sectors.

Experimental: Rock slabs, thin sections, and offcuts were mapped by high-energy synchrotron radiation micro X-ray fluorescence (SR- $\mu$ XRF) for their major and trace element composition at the VESPERS and IDEAS (CLS) beamlines. At IDEAS, samples (up to 10s of cm) were mapped with a $500 \mu \mathrm{m}$ x $500 \mu \mathrm{m}$ beam spot size. At VESPERS samples were mapped using a spot size of $\sim 5 \mu \mathrm{m} \mathrm{x} \sim 5 \mu \mathrm{m}$ and a dwell time of 1 s. All data was analyzed using Peakaboo software.

SR-PXRD patterns, SR-XRF MCA spectra, and XANES spectra were collected on powders of the same samples remotely using the Canadian Macromolecular Crystallography Facility (CMCF) beamline 08BM1 [1] at the CLS. Powdered geological materials were inserted in polyimide capillaries that were mounted in B3S (ALS) style reusable goniometer mounts and placed in a 96-slot SSRL-style cassette. The data collection was performed with the MxDC (Macromolecular Crystallography Data Collector) software. Data was transferred to Western during the session for immediate analysis.

The 08BM-1 beamline endstation setup allows for SR-PXRD, SR-XRF, and XANES data to be collected in series on a single sample. SR-PXRD and SR-XRF data can be collected at energies between $4-18$ $\mathrm{keV}$. In this energy range, XANES spectra can be collected for elements heavier than calcium $(Z>20)$ except for $\mathrm{Nb}(Z=41)$ to $\mathrm{Ag}(Z=47)$. For geological samples, this combination means that mineralogical, geochemical, and chemical speciation and oxidation state information can be gathered rapidly and successively. Phase analysis was performed using Bruker's EVA software [2] with the PDF-2001 
database. XRF data were analyzed using the Peakaboo software package, developed at Western University [3]. XANES data were analyzed using the Athena software program [4].

Results: The rapid analysis of geological and processing materials is not routinely conducted at synchrotron facilities, so we were interested in evaluating the usefulness of existing beamline setups for high throughput analysis. Our goal is to produce large data sets while preserving extremely high-quality X-ray diffraction and X-ray fluorescence data. Examples of these data are shown in Figure 1. We are developing a rigorous sample handling protocol suitable for handling of geological, minerals exploration, processing, waste, and environmental samples. We have evaluated the acquisition parameters that result in robust data collection. We are optimizing data collection for geological samples with different geological matrices for both phase identification as well as for further quantitative Rietveld analysis. We are also developing the methodology for acquiring complimentary SR-XRF data on these same samples and developing a routine analysis using Peakaboo software [3]. Finally, we are collecting oxidation state and chemical speciation for elements of interest such as arsenic and gold. Using a synchrotron source, we were able to collect large volumes of data, incredibly quickly, and with higher quality than is possible with laboratory instruments [5].

\section{References:}

[1] M. Fodje et al, J. Synch. Rad. 21 (2014), p. 633.

[2] Bruker Corporation, EVA Software, www.bruker.com.

[3] N. Sherry et al, Science Studio Project Summary. (2012) http://sciencestudio.net/summary.php.

[4] B. Ravel, M. Newville. J. Synchr. Rad. 12 (2005) p. 537.

[5] Acknowledgements: Research described in this paper was performed at the Canadian Light Source, which is supported by the Canada Foundation for Innovation, Natural Sciences and Engineering Research Council of Canada, the University of Saskatchewan, the Government of Saskatchewan, Western Economic Diversification Canada, the National Research Council Canada, and the Canadian Institutes of Health Research. The authors acknowledge funding from NSERC. We thank M. Fodje, S. Labiuk, R. Feng, and D. Muir for help with data collection and processing.
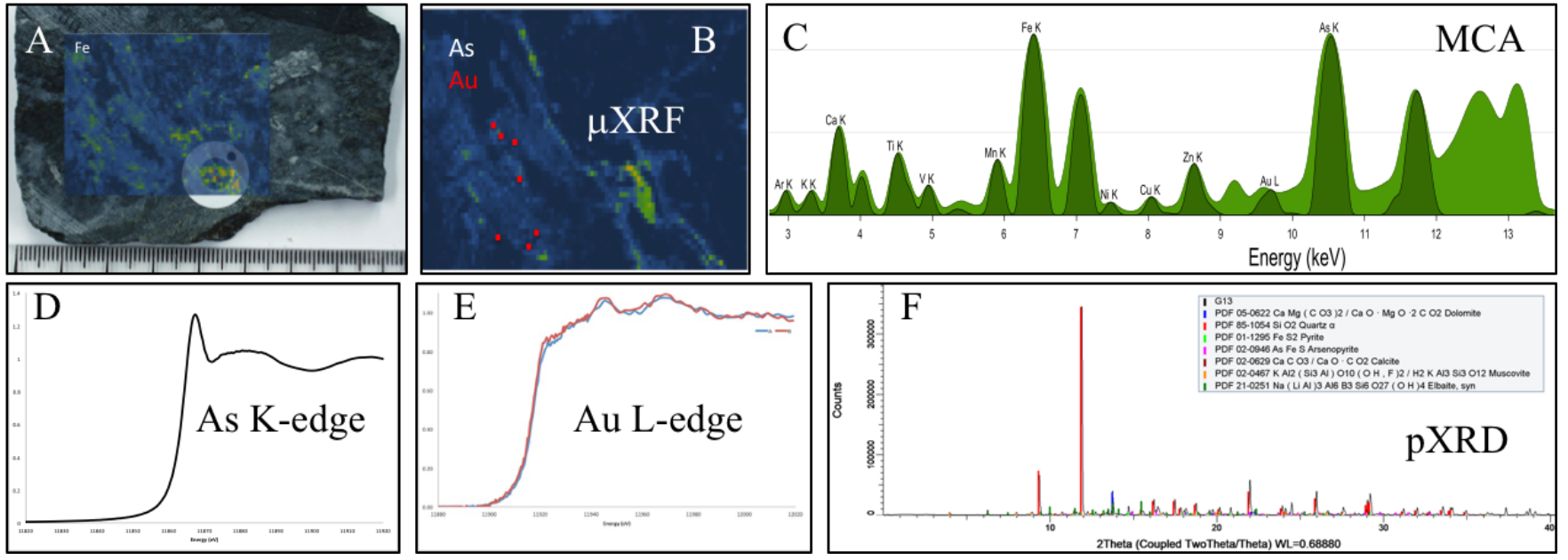

Figure. 1. Examples of complimentary data collected at the IDEAS and CMCF beamlines (CLS). A\&B mXRF maps of sample G-1-3 (overlay in A) showing distribution of Fe, As, and Au. C. Corresponding full map MCA with peak identifications. D\&E As \& Au XANES spectra. E. pXRD diffractogram. 